\title{
Qualité microbiologique du garba, un aliment de rue de Côte d'Ivoire
}

\author{
Adjo Thérèse ANOMAN ${ }^{1,2^{*}}$, Marina KOUSSEMON ${ }^{2}$, Kouadio Ignace KOUASSI ${ }^{3}$ et \\ Yolande AKE ASSI ${ }^{1}$
}

\author{
${ }^{I}$ Ministère de l'Agriculture, LANADA, Laboratoire Central pour l'Hygiène Alimentaire et l'Agro-industrie, 04 \\ BP 612 Abidjan 04, Côte d'Ivoire. \\ ${ }^{2}$ Université Nangui Abrogoua, UFR Sciences et Technologies des Aliments, 02 BP 801 Abidjan 02, \\ Côte d'Ivoire. \\ ${ }^{3}$ Université Nangui Abrogoua, UFR Sciences de la Nature, 02 BP 801 Abidjan 02, Côte d'Ivoire. \\ *Auteur correspondant ; E-mail : anoman_therese@yahoo.fr; Tel : 0022508048034
}

\section{RESUMÉ}

Cette étude s'inscrit dans le cadre de la sécurité sanitaire des aliments de rue notamment le garba qui est très consommé en Côte d'Ivoire. L'objectif de ce travail est l'étude de la qualité microbiologique du garba, un mets ivoirien à base d'attiéké (semoule de manioc) et de thon frit. Pour ce faire, trois cent (300) échantillons de garba ont été prélevés dans quatre communes du district d'Abidjan (Abobo, Cocody, Port bouêt et Yopougon) et analysés. Le dénombrement des différents microorganismes a été effectué selon des méthodes normalisées NF ISO 4833 (2013), NF ISO 4832 (2006), NF ISO 16649-2(2001), NF ISO 15213 (2003), NF ISO 6888-1(2003), ISO 21527(2008) et ISO 7932 (2004) et NF ISO 6579 (2002). Les résultats ont montré la présence de germes aérobies mésophiles, de coliformes thermotolérants notamment Escherichia coli et des levures et moisissures avec des charges minimales respectives de 4,8 $10^{6}$ UFC.g $^{-1}, 9,810^{3}$ UFC.g-1, $^{-1}, 410^{2}$ UFC.g ${ }^{-1}$ et 3,0 $10^{3} \mathrm{UFC}_{\mathrm{g}}{ }^{-1}$. Malgré l'absence de pathogène vrai tels que Salmonella et Bacillus cereus, il ressort que la majorité des échantillons de garba qui proviennent des quatre communes ont une qualité microbiologique non satisfaisante. De tels résultats suggèrent un potentiel risque de toxi-infection alimentaire pour les consommateurs de garba.

(C) 2018 International Formulae Group. All rights reserved.

Mots clefs: Attiéké, thon, germes, microbiologie, aliment de rue.

\section{Microbiological quality of garba, a street food from Côte d'Ivoire}

\begin{abstract}
This study is part of street food safety including garba which is widely consumed in Côte d'Ivoire. The aim of this paper was to study the microbiological quality of garba, an ivoarian dish based on attieké (cassava semolina) and fried tuna. In order to achieve the set goal, a sampling of 300 meals were performed in four communes of the Abidjan district (Abobo, Cocody, Port bouêt and Yopougon) and analyzed. The enumeration of the different microorganisms was carried out according to standardized methods NF ISO 4833 (2013), NF
\end{abstract}


ISO 4832 (2006), NF ISO 16649-2 (2001), NF ISO 15213 (2003), NF ISO 6888-1 (2003), NF ISO 21527 (2008) and ISO 7932 (2004) and NF ISO 6579 (2002). Results showed the presence of mesophilic aerobic bacteria, thermotolerant coliforms, Escherichia coli and yeasts and molds with respective minimum loads of:

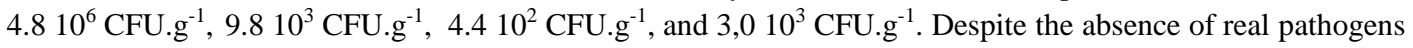
such as Salmonella and Bacillus cereus, it appears that the majority of garba samples from the four communes have unsatisfactory microbiological quality. Such results indicate a potential risk of food infection for garba consumers.

(C) 2018 International Formulae Group. All rights reserved.

Keywords: Attiéké, tuna, germs, microbiology, street food.

\section{INTRODUCTION}

L'alimentation de rue est un phénomène très répandu en Afrique (Neffati et al., 2004). Le terme «aliments de rue» désigne les aliments et les boissons prêts à être consommés, préparés ou vendus par des marchands ambulants spécialement dans les rues et dans les autres lieux publics analogues (FAO, 2007). Ce type d'alimentation, très développé aussi bien en Côte d'Ivoire comme ailleurs, participe quotidiennement aux habitudes alimentaires de millions de citadins dans les grandes villes de ces pays (Ohiokpehai, 2003). Parmi la multitude et la diversité des aliments de rue vendus en Côte d'Ivoire, et particulièrement dans la ville d'Abidjan (capitale économique de la Côte d'Ivoire), le garba semble occuper une place de choix du fait de l'engouement qu'il suscite. C'est l'un des plats locaux les plus appréciés des populations ivoiriennes (FAO, 2012). Le garba est constitué d'attiéké (semoule de manioc) et du thon frit, des oignons, du piment et de la tomate. La vente et la consommation du garba ont connu ces dernières années un essor important à cause de la disponibilité sur le marché ivoirien du poisson communément appelé "faux thon". Ce dernier est constitué par l'ensemble des thons de petite taille, abimés (par les conditions de pêche notamment) ou mal conservés, et ne correspondant pas aux normes techniques des industries de transformation thonières (N'da et al., 2007). Par ailleurs, plusieurs études ont porté sur l'attiéké (Koffi et al., 2004 ; Assanvo et al., 2006 ; Djeni et al., 2010 ; Gbané et al., 2012 ;), par contre celles sur le garba et sa qualité microbiologique sont rares. La qualité du thon et de l'attiéké, de même que celle des légumes frais (oignons, tomates, piments) entrant dans la préparation du garba pourraient certainement constituer une source de contamination microbienne de l'aliment. Ainsi, la présente étude a-t-elle eu pour objectif de déterminer la qualité microbiologique de cet aliment très prisé par les populations ivoiriennes.

\section{MATERIEL ET METHODES \\ Zone et matériel d'étude}

Notre étude a été menée dans la ville d'Abidjan, essentiellement dans 4 communes qui ont constitué notre zone d'étude (Figure 1). Ce sont les communes d'Abobo (Nordest), de Cocody (Centre-est), de Port Bouet (Sud-est) et de Yopougon (Nord-ouest).

L'étude a porté sur 300 échantillons de garba prélevés dans vingt garbadromes (lieux de préparation, de vente et de consommation du garba) dans les communes d'étude à raison de cinq garbadromes par commune.

\section{Méthodes d'analyse microbiologique}

Pour les analyses microbiologiques, des échantillons de garba d'environ $200 \mathrm{~g}$ chacun ont été prélevés au hasard au sein de cinq lieux de vente du garba «garbadromes » dans chacune des communes d'étude. Sur chaque site, trois échantillons (attiéké + thon frit + condiments) ont été prélevés par jour à 8 heures, 12 heures et $17 \mathrm{~h}$. Le prélèvement s'est fait dans les conditions habituelles d'achat, tous les ingrédients, à savoir l'attiéké, le 
poisson et les légumes, servis dans un sachet plastique. Au total, trois cent (300) échantillons ont été prélevés et transportés au laboratoire pour analyse.

Le dénombrement ou la recherche des différents microorganismes a été effectué sur des échantillons composés de l'attiéké, du poisson thon et des condiments broyés et homogénéisés. Une suspension mère a été constituée à partir de $25 \mathrm{~g}$ de cet homogénéisât dans $225 \mathrm{ml}$ d'eau peptonée tamponnée (EPT) à $0,1 \%$ dans un sac Stomatcher stérile.

Les germes aérobies mésophiles ont été dénombrés sur milieu plate count agar (PCA) selon la méthode NF ISO 4833 (2003). Les coliformes thermotolérants ont été dénombrés sur milieu VRBL selon la méthode NF ISO 4832 (2006). Le dénombrement (sur milieu Baird Parker) et l'identification de Staphylococcus aureus ont été réalisés selon la méthode NF ISO 6888-1\&2 (2003). Les anaérobies sulfito-réducteurs ont été dénombrés dans le milieu Gélose au sulfite de fer selon la méthode NF ISO 15213 (2003). La méthode NF ISO 16649-2 a été utilisée pour le dénombrement de Escherichia coli sur milieu TBX (tryptone bile glucuronide) et la méthode ISO 7932 (2004) a été utilisée pour le dénombrement de Bacillus cereus avec le milieu gélose Mossel. Les levures et les moisissures ont été recherchées sur milieu DRBC (Dichloran Rose Bengale
Chloramphenicol) selon la méthode ISO 21527(2008). La recherche des salmonelles a été faite selon la méthode NF ISO 6579 (2002).

Les critères d'appréciation de la qualité des échantillons utilisés sont les normes françaises relatives aux critères auxquels doivent satisfaire les plats cuisinés (règlement CE, 2005).

Ces critères sont présentés dans le Tableau 1. L'interprétation des résultats dérive d'un plan à trois classes et s'effectue de la façon suivante: lorsque les valeurs obtenues sont inférieures aux critères et jusqu'à trois (3) fois le critère, le produit est de qualité microbiologique satisfaisante (QMS). Le produit est de qualité microbiologique acceptable (QMA); lorsque les valeurs obtenues sont entre trois (3) et dix (10) fois le critère. Enfin, la qualité microbiologique est non satisfaisante (QMNS) ; lorsque les valeurs obtenues sont supérieures à dix (10) fois le critère.

L'analyse multiple de la variance (ANOVA) a été utilisée en vue de déterminer la significativité des différences obtenues entre les valeurs des paramètres bactériennes étudiés. Cette significativité est déterminée en comparant la probabilité $P$ associée à la statistique du test de Fischer-Snedecor au seuil théorique de $\alpha=0,05$. Tous les tests ont été effectués grâce au logiciel SAS (SAS, 1999).

Tableau 1 : Critères microbiologiques relatifs aux plats cuisinés (Règlement CE, 2005).

\begin{tabular}{|c|c|c|c|c|c|c|c|}
\hline $\begin{array}{c}\text { GAM } \\
\text { (UFC/g) }\end{array}$ & $\begin{array}{c}\text { C Th } \\
\text { (UFC/g) }\end{array}$ & $\begin{array}{c}\text { E. coli } \\
\text { (UFC/g) }\end{array}$ & $\begin{array}{c}\text { ASR } \\
\text { (UFC/g) }\end{array}$ & $\begin{array}{c}\text { LM } \\
(\mathrm{UFC} / \mathrm{g})\end{array}$ & $\begin{array}{c}\text { S. aureus } \\
\text { (UFC/g) }\end{array}$ & $\begin{array}{c}\text { Salm. } \\
\text { (UFC/25g) }\end{array}$ & $\begin{array}{l}B . \\
\operatorname{Cereus}(\mathrm{UFC} / \mathrm{g})\end{array}$ \\
\hline $3.10^{5}$ & 10 & 10 & 30 & $5.10^{2}$ & $10^{2}$ & Absence & $10^{2}$ \\
\hline
\end{tabular}

GAM: Germes Aérobies Mésophiles; C.Th : Coliformes Thermotolérants; ASR: Anaérobies Sulfito Réducteurs; LM: Levures et Moisissures; Salm: Salmonella. 


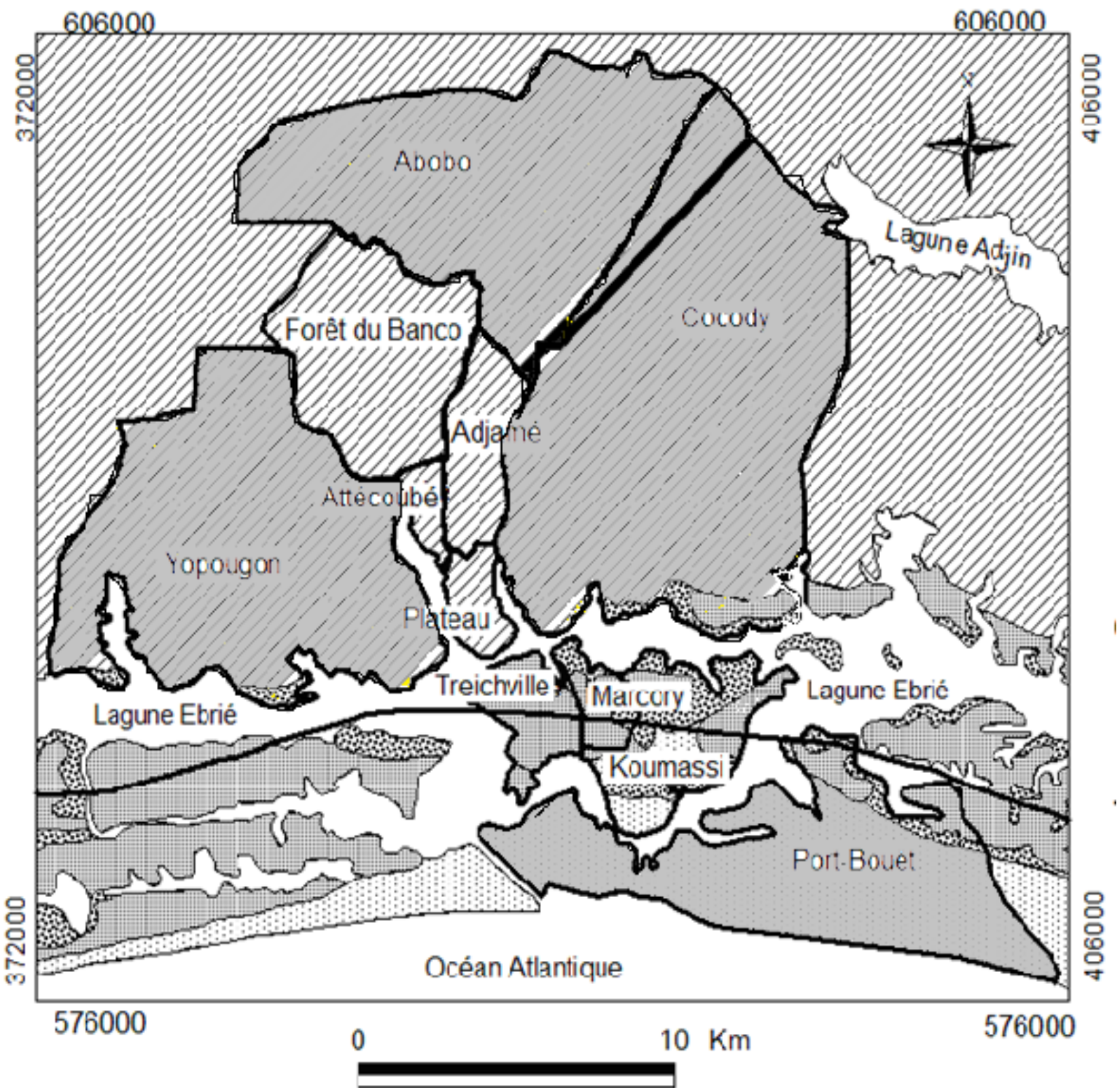

Figure 1 : Carte d'Abidjan indiquant les zones d'étude de la vente de garba. (Source : Ahoussi et al., 2013 modifiée).

\section{RESULTATS}

Dans les échantillons de garba analysés, plusieurs microorganismes tels que des Germes aérobies mésophiles (GAM), des coliformes thermotolérants notamment $E$. coli, des anaérobies sulfito-réducteurs, des levures et moisissures ont été retrouvés (Tableau 2). Quant aux germes pathogènes comme
Bacillus cereus, Staphylococcus aureus et Salmonella, ils n'ont pas été retrouvés dans les échantillons analysés.

Les charges en germes varient en fonction de la nature du germe et de l'heure de prélèvement. Les charges en GAM varient de $4,810^{6}$ à $2,210^{7} \mathrm{UFC} / \mathrm{g}$ et ces charges ne sont pas significativement différentes $(\mathrm{P}>0,05)$ 
d'une heure de prélèvement à une autre à l'exception de ceux des communes de Cocody, Port Bouêt et Yopougon où les prélèvements de $17 \mathrm{~h}$ sont significativement différents $(\mathrm{P}<$ $0,05)$.

Les charges en coliformes thermotolérants des différents échantillons se situent entre $9,810^{3}$ et $1,210^{5} \mathrm{UFC} / \mathrm{g}$. E. coli n'a pas été dénombrés à tout moment de la journée et selon les heures de prélèvement, les charges en $E$. coli varient de $4,410^{2}$ à $6,010^{3}$ UFC/g. En effet, de façon générale les charges en E. coli sont importantes dans les échantillons prélevés à $12 \mathrm{~h}$ et à $17 \mathrm{~h}$, les ASR ne sont présents que dans les échantillons prélevés à $17 \mathrm{~h}$. Les charges en levures moisissures varient de $3,010^{3}$ à $7,510^{3} \mathrm{UFC} / \mathrm{g}$ et sont significativement différentes d'une heure de prélèvement à une autre et l'intérieur de chaque commune. Les échantillons de garba analysés sont majoritairement de qualité microbiologique non satisfaisante. En effet, au regard des critères microbiologiques retenus, $98,67 \%, 100 \%, 96 \%$ et $97,34 \%$ des échantillons prélevés respectivement dans les communes d'Abobo, de Port-Bouet de Yopougon et de Cocody sont de qualité microbiologique non satisfaisante (Figure 2).

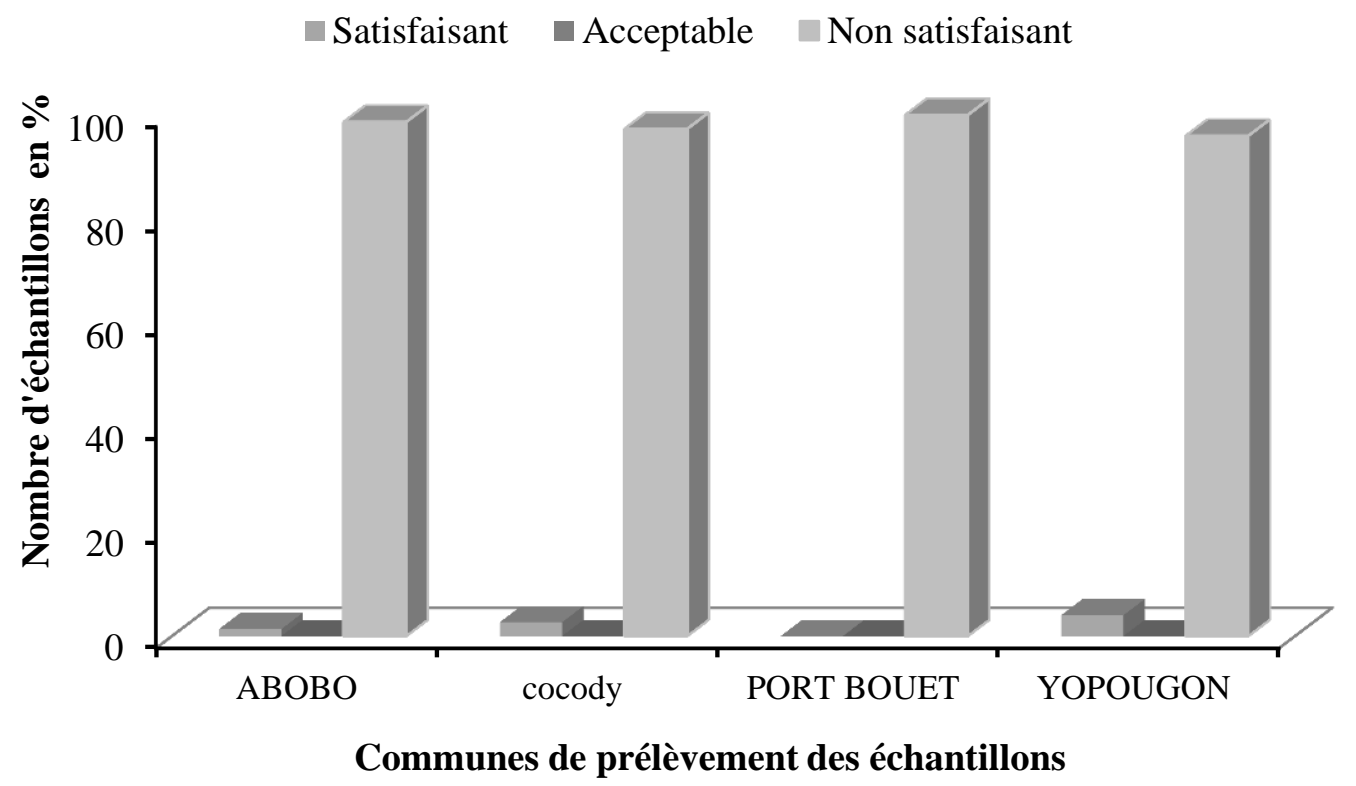

Figure 2: Qualité microbiologique du garba dans les différentes communes. 
Tableau 2 : Charges bactériennes (UFC/g) des échantillons de garba.

\begin{tabular}{|c|c|c|c|c|c|c|c|c|c|c|c|c|}
\hline & \multicolumn{3}{|c|}{ Abobo } & \multicolumn{3}{|c|}{ Cocody } & \multicolumn{3}{|c|}{ Port-Bouêt } & \multicolumn{3}{|c|}{ Yopougon } \\
\hline & 8 heures & 12 heures & 17 heures & 8 heures & 12 heures & 17 heures & 8 heures & 12 heures & 17 heures & 8 heures & 12 heures & 17 heures \\
\hline GAM & $\begin{array}{c}2,210^{7} \pm 3,7 \\
10^{7 \mathrm{a}}\end{array}$ & $\begin{array}{c}1,710^{7} \pm 1,2 \\
10^{7 a}\end{array}$ & $\begin{array}{c}1,310^{7} \pm 1,2 \\
10^{7 \mathrm{a}}\end{array}$ & $\begin{array}{c}7,810^{6} \pm 9,7 \\
10^{6 \mathrm{~b}}\end{array}$ & $\begin{array}{c}7,110^{6} \pm 9,2 \\
10^{6 \mathrm{~b}}\end{array}$ & $\begin{array}{c}1,710^{7} \pm 1,3 \\
10^{7 \mathrm{a}}\end{array}$ & $\begin{array}{c}7,510^{6} \pm 1,6 \\
10^{7 \mathrm{~b}}\end{array}$ & $\begin{array}{l}4,810^{6} \pm \\
5,110^{6 \mathrm{~b}}\end{array}$ & $1,110^{7} \pm 1,210^{7 \mathrm{a}}$ & $\begin{array}{c}1,4 \\
10^{7} \pm 1,310^{7 \mathrm{ab}}\end{array}$ & $\begin{array}{c}9,910^{6} \pm 1,3 \\
10^{7 \mathrm{~b}}\end{array}$ & $\begin{array}{c}1,810^{7} \pm 1,2 \\
10^{7 \mathrm{a}}\end{array}$ \\
\hline Cth & $\begin{array}{c}1,210^{5} \pm 5,1 \\
10^{4 a}\end{array}$ & $\begin{array}{c}1,110^{5} \pm 6,1 \\
10^{4 a}\end{array}$ & $\begin{array}{c}7,710^{4} \pm 7,1 \\
10^{4 a}\end{array}$ & $\begin{array}{c}7,510^{4} \pm 1,1 \\
10^{5 \mathrm{~b}}\end{array}$ & $\begin{array}{c}4,610^{4} \pm 6,0 \\
10^{4 \mathrm{~b}}\end{array}$ & $\begin{array}{c}1,110^{5} \pm 1,2 \\
10^{5 \mathrm{a}}\end{array}$ & $\begin{array}{c}9,810^{3} \pm 6,3 \\
10^{3 \mathrm{c}}\end{array}$ & $\begin{array}{l}1,210^{5} \pm \\
7,610^{4 a}\end{array}$ & $7,110^{4} \pm 6,410^{4 a}$ & $\begin{array}{c}7,8 \\
10^{4} \pm 7,110^{\mathrm{ab}}\end{array}$ & $\begin{array}{c}9,010^{4} \pm 7,0 \\
10^{4 a}\end{array}$ & $\begin{array}{c}1,010^{5} \pm 6,7 \\
10^{4 a}\end{array}$ \\
\hline $\begin{array}{c}E . \\
\text { coli }\end{array}$ & $\begin{array}{c}4,410^{2} \pm 1,6 \\
10^{3 \mathrm{a}}\end{array}$ & $\begin{array}{c}6,010^{3} \pm 2,1 \\
10^{4 \mathrm{a}}\end{array}$ & $\begin{array}{c}3,010^{3} \pm 1,5 \\
10^{4 \mathrm{ab}}\end{array}$ & 0 & $\begin{array}{l}9,610^{2} \pm \\
4,810^{3 \mathrm{a}}\end{array}$ & 0 & 0 & $\begin{array}{l}4,910^{3} \pm \\
2,410^{4 a}\end{array}$ & $1,1 \quad 10^{4} \pm 2,510^{4 a}$ & $\begin{array}{c}6,010^{3} \pm 2,1 \\
10^{4 a}\end{array}$ & 0 & $\begin{array}{c}7,410^{2} \pm 2,5 \\
10^{3 \mathrm{~b}}\end{array}$ \\
\hline $\mathbf{L M}$ & $\begin{array}{c}7,410^{3} \pm 4,2 \\
10^{3 \mathrm{a}}\end{array}$ & $\begin{array}{c}7,110^{3} \pm 4,4 \\
10^{3 \mathrm{a}}\end{array}$ & $\begin{array}{c}3,010^{3} \pm 3,4 \\
10^{3 \mathrm{c}}\end{array}$ & $\begin{array}{c}5,510^{3} \pm 3,3 \\
10^{3 \mathrm{~b}}\end{array}$ & $\begin{array}{c}5,010^{3} \pm 3,3 \\
10^{3 \mathrm{~b}}\end{array}$ & $\begin{array}{c}6,410^{3} \pm 4,0 \\
10^{3 \mathrm{ab}}\end{array}$ & $\begin{array}{c}3,510^{3} \pm 3,2 \\
10^{3 \mathrm{c}}\end{array}$ & $\begin{array}{l}6,010^{3} \pm \\
1,510^{4 a b}\end{array}$ & $\begin{array}{c}4,010^{3} \pm 3,2 \\
10^{3 \mathrm{bc}}\end{array}$ & $\begin{array}{c}6,410^{3} \pm 4,3 \\
10^{3 \mathrm{ab}}\end{array}$ & $\begin{array}{c}3,510^{3} \pm 4,7 \\
10^{3 \mathrm{c}}\end{array}$ & $\begin{array}{c}7,510^{3} \pm 5,2 \\
10^{1 \mathrm{a}}\end{array}$ \\
\hline ASR* & 0 & 0 & 10 & 0 & 0 & 20 & 0 & 0 & 10 & 0 & 0 & 10 \\
\hline
\end{tabular}

* pour les ASR, les valeurs des charges obtenues à 17 heures représentent la valeur moyenne des échantillons positifs

GAM : Germes Aérobies Mésophiles. Cth ; Coliformes thermotolérants, E Coli : Escherichia coli ; LM: Levures-Moisissures, ASR : Anaérobies Sulfito-Réducteurs

Les valeurs portant la même lettre sur une ligne ne sont pas significativement différentes au seuil de 5\%. 


\section{DISCUSSION}

Les charges microbiennes des germes retrouvés dans le garba (GAM, coliformes thermotolérants, E. coli, levures et moisissures) sont supérieures à celles tolérées pour les plats cuisinés. Les levures et moisissures sont particulièrement abondantes dans les échantillons de garba analysés.

La présence de tous ces germes pourrait s'expliquer par les conditions de la pratique de l'activité de production et de commercialisation du garba. En effet, tous les germes retrouvés dans les échantillons pourraient provenir de l'environnement de vente (proximité des routes, vente à ciel ouvert...), des vendeurs eux-mêmes (mains et vêtements sales) ou encore des éléments intervenant dans la préparation du garba. Certaines études ont, en effet, déjà indiqué la présence de germes aérobies mésophiles, des coliformes totaux et des coliformes thermotolérants dans l'attiéké (Koffi et al., 2004 ; Kouamé et al., 2012). Les légumes frais, à savoir la tomate, le piment et l'oignon peuvent également constituer des apports de germes s'ils ne sont pas frais et/ou, pas correctement lavés. Les légumes sont, en effet, couramment impliqués dans des cas de toxi-infection alimentaire (Belomaria et al., 2007). Le poisson peut cependant être peu incriminé car il est frit et servi généralement très chaud. En comparaison aux critères retenus pour les plats cuisinés, les importantes charges microbiennes retrouvées dans les échantillons analysés ont permis de noter la qualité microbiologique non satisfaisante de 98\% des échantillons analysés par rapport aux critères microbiologiques retenus. Ces résultats sont confortés par ceux de (Barro et al., 2005 ; Elmarnissi et al., 2012 ; Oluwafemi, 2013) qui ont travaillé sur différents aliments de rue de quelques régions du Maroc, de Ouagadougou et du Nigeria respectivement et qui ont trouvé des résultats similaires. Ces auteurs ont, en effet, trouvé que des échantillons analysés étaient de qualité microbiologique non satisfaisante. Une telle situation laisse supposer un risque sur la santé des consommateurs malgré l'absence de pathogènes vrais tels que $S$. aureus, Salmonella et Bacillus cereus dans les échantillons.

\section{Conclusion}

L'objectif de ce travail est l'étude de la qualité microbiologique du garba, un mets ivoirien à base d'attiéké (semoule de manioc) et de thon frit. Les charges microbiennes des germes retrouvés dans les échantillons de garba analysés indiquent que la majorité des échantillons est de qualité non satisfaisante par rapport aux critères microbiologiques. Ces résultats suggèrent que la consommation de cet aliment pourrait représenter un risque pour la santé des consommateurs. Il est donc nécessaire d'améliorer la qualité hygiénique du garba pour assurer une meilleure sécurité des consommateurs. De ce fait nous préconisons une sensibilisation aux bonnes pratiques d'hygiènes et de fabrication aux acteurs de la filière.

\section{CONFLIT D'INTERETS}

Les auteurs déclarent qu'il n'y a aucun conflit d'intérêts pour cet article.

\section{CONTRIBUTIONS DES AUTEURS}

ATA a procédé à la collecte et à l'analyse des échantillons puis à la rédaction et à la correction de l'article. MK a contribué à la rédaction et à la relecture de cet article. KIK a participé au traitement statistique des résultats. YAA a contribué à la relecture de l'article.

\section{REMERCIEMENTS}

Nos remerciements vont à l'endroit de la direction du Laboratoire National d'Appui au Développement Agricole (LANADA) qui a permis la réalisation de ce travail dans une de ces structure technique le laboratoire central pour l'Hygiène Alimentaire et l'AgroIndustrie (LCHAI).

\section{RÉFÉRENCES}

Ahoussi KE, Loko S, Koffi B, Soro G, Oga YMS, Soro N. 2013. Evolution spatiotemporelle des teneurs en nitrate des eaux souterraines de la ville d'Abidjan (Côte d'Ivoire). Int. J. Pure App. Biosc., 1(3) : 45-60.

Assanvo JB, Agbo GN, Behi YEN, Farah Z. 2006. Microflora of traditional starter made from cassava for attiéké production in Dabou (Côte d'Ivoire). Food Control., 
17(1):

$37-41$.

DOI:

10.1016/j.foodcont.2004.08.006.

Bachir El B; Souad B; Nesma N; Issad N; Abdelghani M; Abdelmajid S, Mohamed E. 2014. Les intoxications alimentaires isolées dans la province d'Errachidia, Maroc. Int. J. Inn. Appl. Stu., 8(2):697704.

Barro N; Lassina S; Marc CT; Cheik AT, Traore AS. 2005. Les principaux agents du péril identifiés dans les aliments de rue et ceux des cantines et leur prévalence en milieu hospitalier, Maitrise des procédés en vue d'améliorer la qualité et la sécurité des aliments, utilisation des OGM, Analyse des risques en agroalimentaire Ouagadougou, 8-11.

Belomaria M, Ahami OAT, Aboussaleh Y, Elbouhali1 B, Cherrah Y, Soulaymani A. 2007. Origine environnementale des intoxications alimentaires collectives au Maroc : Cas de la région du Ghrab chrarda BniHssen. Antropo., 14: 83-88.

Benlarabi S, Semlali I, Eloufir G, Badri M, Souaymani BR. 2006. Les toxi infections alimentaires collectives: données du centre Anti poison et de pharmacovigilance du Maroc. Premier congrès National de la Société Marocaine de Toxicologie Clinique et Analytique. Institut National de l'administration Sanitaire (INas), Rabat, Maroc.

Djéni NT, N'guessan KF, Toka DM, Kouamé KA, Dje KM. 2011. Quality of attieke (a fermented cassava product) from the three main processing zones in Côte d'Ivoire. Food Res Inter., 44(1): 410416.

DOI:

10.1016/j.foodres.2010.09.032

Elmarnissi B, Bennani L, El Oulali Lalami, Aobouch M, Belkhou R. 2012. Contribution à l'étude de la qualité microbiologique de denrées alimentaires commercialisées à Fes-Boulemane. Rev. Microbiol. Ind. San. Environn., 6(1) : 98117.

FAO. 2007. Food Agricultural Organization of the United Nation - FAO Bonne pratique d'hygiène dans la préparation et la vente des aliments de rue en Afrique. Rome, Italie, 188p.
FAO. 2012. Food Agricultural Organization of the United Nation - FAO Street food vending in West Africa cities: Potential and challenges; Rome.

Gbané M, Coulibaly A, Niaka KPV, Adou M. 2012. Composition physico-chimique de deux mets de rue (le plat d'attiéké et le garba) vendu à Abidjan. A. Biomed., 17(3): 26-33.

Koffi LB, Djedji CE, Kamenan. A. 2004. Taux irréductible d'acide cyanhydrique et qualité microbiologique de l'attiéké produit dans la région d'Abidjan. Agron. Afr., 16(3): 11-19. DOI : http /dx.doi.org/10.4314/aga.v16i3.1649.

Kouame AK, Djeni TN, N'guessan FN, Dje MK. 2012. Postprocessing microflora of commercial attieke (afermented cassav aproduct) produced in the south of Côte d'Ivoire. Lett. Appl. Microbiol., 56(1): 44-50. DOI: https://doi.org /10.1111/1am.12014.

N'da K, Dedo GR, Alain H. 2007. Le débarquement des faux thons ou faux poissons au port de pêche d'Abidjan : phénomène en résurgence dans les données ICCAT en Côte d'Ivoire. Col. Vol. Sci. Pap. ICCAT, 60(1): 180-184.

Neffati L, Ridha H, Kolsteren P, Hilderbrand K. 2004. L'alimentation de rue chez l'écolier dans une région du nord de la Tunisie. Cah. Santé, 14(1): 43-48.

Ohiokpehai O. 2003. Nutritional aspects of street foods in Botswana. Pakistan J. Nutr., 2(2): 76-81. DOI: 10.3923/pjn.2003.76.81.

Oluwafemi F, Akisanya E, Odeniyi K, Salami W, Sharomi T. 2013. Microbiological quality of street-vended foods and readyto-Eat vegetables in some Nigeria Cities. Afri. J. Biomed. Res., 16(3): 163-166.

Règlement (CE) N²073/2005 de la commission des communautés européennes, du 15 Novembre 2005, concernant les critères microbiologiques applicables aux denrées alimentaires. Journal Officiel de l'Union Européenne.18p.

SAS, 1999. SAS/ETS User's Guide, vers 6, 4th ed SAS Inst. Cary, NC: 501. 